\section{Deaths from pesticide poisoning: a global response}

\author{
J. M. BERTOLOTE, A. FLEISCHMANN, M. EDDLESTON and D. GUNNELL
}

with millions of cases of pesticide poisoning, hundreds of thousands of which result in deaths each year in low- and middle-income countries. There is an urgent need for immediate action.

\begin{abstract}
Summary Self-poisoning with pesticides accounts for about a third of all suicides worldwide. To tackle this problem, the World Health Organization announced a global public health initiative in the second half of 2005. Planned approaches were to range from government regulatory action to the development of new treatments for pesticide poisoning. With broad-based support, this strategy should have a major impact on the global burden of suicide.
\end{abstract}

\section{Declaration of interest D.G. and}

M.E. are on the Scientific Advisory Group of a Syngenta-funded study to assess the toxicity of a new formulation of paraquat; they have received travel expenses to attend research group meetings.

Suicide is an important cause of premature mortality, accounting for an estimated 877000 deaths every year (World Health Organization, 2003). In many agricultural communities of low- and middle-income countries, pesticide self-poisoning accounts for a large proportion of these deaths (Gunnell \& Eddleston, 2003). In 1985, using data from Sri Lanka, Jeyaratnam estimated that there were approximately 2.9 million cases of acute pesticide poisoning, resulting in about 220000 deaths each year in the developing world (Jeyaratnam, 1985a). More recently, according to the finding that pesticide ingestion accounted for over $60 \%$ of suicides in many rural areas of China and South East Asia, Gunnell \& Eddleston (2003) estimated that there may be as many as 300000 deaths each year from intentional poisoning (i.e. suicide by ingestion of pesticides) in these regions alone. This makes it likely that pesticide poisoning is the most frequently used method of suicide worldwide.
The research and policy attention that pesticide poisoning has received has been relatively small in comparison with the magnitude of the problem (Gunnell \& Eddleston, 2003; Buckley et al, 2004). Jeyaratnam (1985a) suggested that part of the reason why pesticide poisoning had not received the global attention it deserved was the lack of scientific evidence concerning the extent of the problem. Even though a global estimate is still not possible today, because of the lack of large-scale, rigorous surveillance data, evidence is growing that the burden of pesticide poisoning has by no means been underestimated. Although data from lowincome countries in South America are less comprehensive than from China and South-East Asia, evidence is emerging that pesticide poisoning is equally important in such settings. In Brazil, for example, the high suicide rates in tobacco growing regions may be owing to the wide use and availability of pesticides (Csillag, 1996). Furthermore, pesticides accounted for $18 \%$ of male and $7 \%$ of female hospital admissions for self-harm in Campinas, Brazil, in 2002-2004 (Fleischmann et al, 2005). In Southern Trinidad, over $80 \%$ of suicides in one rural area were by pesticide poisoning (Hutchinson et al, 1999). In Suriname, a high proportion of both fatal $(55 \%)$ and non-fatal $(44 \%)$ episodes of suicidal behaviour involved pesticides (Graafsma et al, 2006). In Africa, data from Zimbabwe show rising levels of admissions for organophosphate selfpoisoning; self-poisoning accounted for about three-quarters of the approximately 200 admissions in one study (Dong \& Simon, 2001). More recently, data from Malawi implicated pesticide self-poisoning in almost $80 \%$ of suicides (Dzamalala et al, 2006).

Despite the problems in estimating the global burden of pesticide poisoning, we may safely assume that we are confronted

\section{APPROACHES TO SUICIDE PREVENTION}

Two of the major components of current international suicide prevention strategies are the improved recognition and treatment of mental illness and restricting access to commonly used methods of suicide (World Health Organization, 2004).

Mental disorders are found less frequently in suicides in Asian than in Western countries, possibly because a greater proportion of deaths in Asia result from the use of highly lethal pesticides in impulsive acts of self-harm. Nevertheless, mental illness is present in a significant proportion of Asian suicides (Phillips et al, 2002) and is thought to play an important role in suicidal behaviours, underlining the role of psychiatrists and other mental health workers in these settings.

Epidemiological evidence demonstrates that restricting access to commonly used methods of suicide may lead to reductions in overall as well as method-specific suicide rates. The potential impact of changing the availability of pesticides on both pesticide poisoning and overall suicide numbers has been demonstrated in Western Samoa (Bowles, 1995). Furthermore, the benefits of restricting pesticide use to those compounds least harmful to humans in self-poisoning is demonstrated by the widely differing case fatality rates associated with different pesticides - from $>60 \%$ for paraquat (Hettiarachchi \& Kodithuwakku, 1989) to $<10 \%$ for several other products (Eddleston, 2000; Eddleston et al, 2005). Likewise, just as the development and widespread use of $\mathrm{N}$-acetyl cysteine had a dramatic effect on trends in paracetamol mortality in England and Wales (Flanagan \& Rooney, 2002), so too may the development of new antidotes to pesticide self-poisoning reduce suicides in low- and middle-income countries. International research in this area should be a global priority (Buckley et al, 2004). However, to date, the global response to the problem of self-poisoning with pesticides has been poor. 


\section{AN INTERSECTOR AL GLOBAL PUBLIC HEALTH INITIATIVE}

In collaboration with other relevant United Nations agencies, governments, academic institutions, non-governmental organisations and interested parties, the World Health Organization has announced an intersectoral global public health initiative with the overall goal of reducing morbidity and mortality related to pesticide poisoning, adhering to a strategy of concerted action at various levels. The five objectives of the programme are to:

(a) review and recommend improved pesticide regulatory policies;

(b) implement sustainable epidemiological surveillance and monitoring of pesticide poisoning in clinical settings and communities;

(c) improve the medical management and mental healthcare of people with pesticide poisoning in healthcare facilities at different levels;

(d) provide training in the safe handling of pesticides and identification and management of pesticide poisoning at different sectors and levels;

(e) develop or strengthen community programmes that minimise risks of intentional and unintentional pesticide poisoning.

There are three components to the World Health Organization strategy. First, to establish sentinel projects in selected countries with a high burden of intentional and unintentional pesticide poisoning. Second, to establish regional centres, associated with the sentinel project sites, for the prevention and management of pesticide poisoning and for enhanced surveillance, training and community action. Third, to disseminate information to governments, decision makers and the public at large about insights, experience, and lessons learnt from research, formulated as recommendations to guide global policy and local action for the prevention and management of pesticide poisoning.

When suicidal behaviour plays an important role in non-fatal and fatal selfpoisoning by pesticides, it is key that relevant preventive measures are anchored in national suicide prevention programmes; these, unfortunately, mostly do not exist as yet in low- and middle-income countries. To this end, the World Health Organization has started to work with governments

J. M. BERTOLOTE, MD, A. FLEISCHMANN, PhD, Department of Mental Health and Substance Abuse, World Health Organization, Geneva, Switzerland; M. EDDLESTON, Scottish Poisons Information Bureau, Royal Infirmary, Edinburgh; D. GUNNELL, PhD, Departemnt of Social Medicine, University of Bristol, Bristol, UK

Correspondence: José M. Ber tolote, Department of Mental Health and Substance Abuse, World Health Organization, 20 avenue Appia, CH-I2II Geneva 27, Switzerland. Email: bertolotej@who.int

(First received 16 December 2005, final revision 3 April, 2006, accepted 2 May 2006)

worldwide to raise awareness of the importance and magnitude of suicidal behaviours and to provide technical assistance in the development and implementation of national strategies for suicide prevention.

It is crucial that governments ratify and implement the hazardous chemicals and wastes conventions (i.e. the Basel, Rotterdam, and Stockholm Conventions) which cover key elements of the management of hazardous chemicals (see http://www.basel.int; http://www.pic.int; http://www.pops.int). Whereas this first crucial step has been taken by many countries, the implementation poses major challenges, such as the shortage of funds, the lack of expertise, human resources and training, the need for advanced technologies and equipment, the limited database systems available and the lack of public awareness. Konradsen et al (2005) stressed the need for these conventions not only to propose actions such as phasing out the most toxic chemicals, promoting alternative pest management strategies, or improving safe storage, but also to explicitly include self-poisonings in risk assessments and to deal directly with the subject of self-harm.

\section{CLOSING REMARKS}

The available data suggest that acute pesticide poisoning has been a major problem in low- and middle-income countries for many years and that it should be a major public health concern today. Jeyaratnam $(1985 b)$ argued over 20 years ago that most research studies and publications on the impact of pesticides on health concentrated on aspects relevant to the industrialised world, and that the industrialised countries have in fact been able to successfully control pesticide poisoning.

Although little has been done in lowand middle-income countries, there is encouraging work in progress. Pilot studies are under way in some areas of China and
Sri Lanka to assess the effects on suicide and suicide attempts in rural areas of the adoption by farmers of a double-locked box where pesticides are stored. In Sri Lanka, the non-governmental organisation Sumithrayo and other groups are active in evaluating interventions of this sort. Early results from one group working on the feasibility of double-locked boxes in Sri Lanka suggest that this is a practical approach to pursue, if developed in collaboration with local communities, signalling the importance of large-scale evaluations (F. Konradsen, personal communication, 2006). With creativity, willingness and appropriate funds much can be done to prevent this unnecessary loss of life.

Considerable work is still required to determine the appropriate balance between public health approaches to reduce pesticide poisoning and the need of the agricultural sector for crop protection products.

Action has to be effective at least in the areas of pesticide policy, pesticide information, mental healthcare, clinical management of pesticide intoxication and restricting hazardous exposure to pesticides. In view of the complexity and novelty of many of these areas, a solid yet feasible research programme has to back them. This will only succeed if broad intersectoral and financial support is made available involving the governmental and the private sector, including relevant nongovernmental organisations and agencies of the United Nations.

\section{REFERENCES}

Bowles, J. R. (1995) Suicide in Western Samoa: an example of a suicide prevention program in a developing country. In Preventive Strategies on Suicide (eds R. F.W. Diekstra, et al), pp. 173-206. Leiden: Brill.

Buckley, N. A., Roberts, D., Eddleston, M. (2004)

Overcoming global apathy in research on

organophosphate poisoning. BMJ, 329, 1231-1233.

Csillag, C. (1996) Brazil's soaring suicide rate revealed. Lancet, 348, 1651 . 
Dong, X. \& Simon, M. A. (200I) The epidemiology of organophosphate poisoning in urban Zimbabwe from 1995 to 2000. International journal of Occupational and Environmental Health, 7, 333-338.

Dzamalala, C. P., Milner, D. A. \& Liomba, N. G.

(2006) Suicide in Blantyre, Malawi (2000-2003). Journal of Clinical Forensic Medicine, 13, 65-69.

Eddleston, M. (2000) Patterns and problems of deliberate self-poisoning in the developing world. QJM: Monthly Journal of the Association of Physicians, 93 7|5-73!.

\section{Eddleston, M., Eyer, P., Worek, F., et al (2005)}

Differences between organophosphorus insecticides in human self-poisoning: a prospective cohort study. Lancet, 366, 1452-1459.

Flanagan, R. J. \& Rooney, C. (2002) Reducing acute poisoning deaths. Forensic Science International, 128, 3-19.

Fleischmann, A., Bertolote, J. M., de Leo, D., et al (2005) Characteristics of attempted suicides seen in emergency-care settings of general hospitals in eight low- and middle-income countries. Psychological Medicine, 35, 1467-1474.

Graafsma, T., Kerkhof, A., Gibson, D., et al (2006) High rates of suicide and attempted suicide using pesticides in Nickerie, Suriname, South America. Crisis, 27, in press.

Gunnell, D. \& Eddleston, M. (2003) Suicide by intentional ingestion of pesticides: a continuing tragedy in developing countries. International Journal of Epidemiology, 32, 902-909.

Hettiarachchi, J. \& Kodithuwakku, G. C. S. (1989) Pattern of poisoning in rural Sri Lanka. International Journal of Epidemiology, 18, 418-422.

Hutchinson, G., Daisley, H., Simeon, D., et al (1999) High rates of paraquat-induced suicide in southern Trinidad. Suicide and Life Threatening Behavior, 29, |86-19|.
Jeyaratnam, J. (1985a) Health problems of pesticide usage in the third world. British Journal of Industrial Medicine, 42, 505-506.

Jeyaratnam, J. (1985b) 1984 and occupational health in developing countries. Scandinavian Journal of Work and Environmental Health, II, 229-234.

Konradsen, F., van der Hoek, W., Gunnell, D., et al (2005) Missing deaths from pesticide self-poisoning in the IFCS Forum IV. Bulletin of the World Health Organization, 83, 157-158.

Phillips, M. R., Yang, G., Zhang, Y., et al (2002) Risk factors for suicide in China: a national casecontrol psychological autopsy study. Lancet, $\mathbf{3 6 0}$, 1728-1736.

World Health Organization (2003) The World Health Report 2003; Shaping the Future. Geneva: WHO.

World Health Organization (2004) The Prevention of Mental Disorders: Effective Interventions and Policy Options. Geneva: WHO 